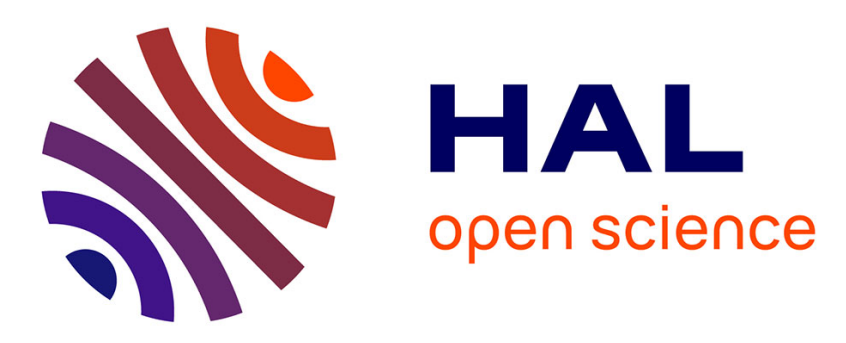

\title{
Modelling and Control of a Complex Buoyancy-Driven Airship
}

Xiaotao . Wu, Claude Moog, Luis Alejandro Márquez-Martínez, Yueming Hu

\section{To cite this version:}

Xiaotao . Wu, Claude Moog, Luis Alejandro Márquez-Martínez, Yueming Hu. Modelling and Control of a Complex Buoyancy-Driven Airship. 8th IFAC Symposium on Nonlinear Control Systems, Sep 2010, Bologna, Italy. pp.1134-1139. hal-00584873

\section{HAL Id: hal-00584873 \\ https://hal.science/hal-00584873}

Submitted on 11 Apr 2011

HAL is a multi-disciplinary open access archive for the deposit and dissemination of scientific research documents, whether they are published or not. The documents may come from teaching and research institutions in France or abroad, or from public or private research centers.
L'archive ouverte pluridisciplinaire HAL, est destinée au dépôt et à la diffusion de documents scientifiques de niveau recherche, publiés ou non, émanant des établissements d'enseignement et de recherche français ou étrangers, des laboratoires publics ou privés. 


\title{
Modelling and Control of a Complex Buoyancy-Driven Airship ${ }^{\star}$
}

\author{
Xiaotao WU ${ }^{*, * * *}$ Claude H. MOOG ${ }^{*}$ L.A. Marquez-Martinez ${ }^{* *}$ \\ Yueming $\mathbf{H U}^{* * *}$ \\ * Institut de Recherche en Communications et Cybernétique de Nantes, 1 rue \\ de la Nö̈, 44321, Nantes, France(Tel: 0033-240376927; e-mail: \\ $\{$ xiaotao.wu\}\{claude.moog $\} @ i r c c y n . e c-n a n t e s . f r)$ \\ ${ }^{* *}$ Centro de Investigación Cientfica y de Educación Superior de Ensenada, \\ Baja California, Mexico(e-mail:Imarquez@cicese.mx) \\ *** South China University of Technology, Guangzhou, China(e-mail: \\ auymhu@scut.edu.cn)
}

\begin{abstract}
The general model for a new generation airship is introduced from the model of an elementary mechanical system which embodies the core of the problem to more complex. It is shown that the basic properties of a suitable two degree of freedom mechanical system are instrumental for the analysis and synthesis of advanced airships. It is shown that the control of the airship mechanical system yields suitable approximations for the control of the airship subject to aerodynamic forces.
\end{abstract}

Keywords: autonomous vehicles; aircraft control; airship; feedback linearization; Minimum-phase systems

\section{INTRODUCTION}

A new generation of airships is considered. New energy saving actuators are introduced which consist in a moving ballast and a tuning of buoyancy Purandare [2007], Wu, Moog et Hu. [2009], $\mathrm{Wu}$, Moog, Martinez et Hu. [2009]. The scheme is inspired from underwater gliders which use similar features to minimize the use of standard propulsors Leonard et al. [2001].

An unmanned autonomous airship equipped with standard propulsors was introduced in Gomes [1998] for the first time, and it is the standard model of the main stream in the airship research. Inspired by underwater gliders Leonard et al. [2001], Purandare [2007] designed a new-kind of airship whose main properties are mentioned above. Wu, Moog et $\mathrm{Hu}$. [2009] used an aeronautical way to derive a complete model of this new generation airship and designed a LQR controller for its stabilization. Wu, Moog, Martinez et Hu. [2009] designed a nonlinear controller based on input-output linearization of the pitch angle. With the controller in $\mathrm{Wu}, \mathrm{Moog}$, Martinez et $\mathrm{Hu}$. [2009], the state variables are critically stable.

As done in Wu, Moog, Martinez et Hu. [2009], the airship is considered restricted to a planar motion and subject to the single control input by moving ballast in this paper.

The goals in this paper are:

(1) Display the fundamental and elementary structure hidden in the complex full model of the airship.

(2) Show how the use of this fundamental structure is instrumental to derive new control schemes!

To reach goal (1), the modelling of the airship is done first from simple special case (with a fixed center of volume) and then to more complex and complete cases. In this way essential

\footnotetext{
$\star$ This work is supported by CSC
}

properties hidden in the model are highlighted and applied to simplify the control problem of the most complete model. The most elementary special case is a simple two degree of freedom mechanical system whose properties are claimed to invariant under the future complexification of the system. Also based on this elementary special case, we set up the complete model through dropping restrictions and adding external forces. For the goal (2), the most elementary system is controlled by computing a suitable dummy output function which defines a minimum phase system and whose feedback linearization achieves maximal linearization of the considered system, which instructs the way to design the controller for the complete model.

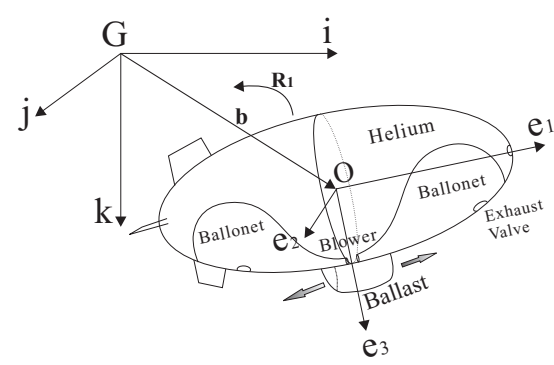

Fig. 1. Structure of a Buoyancy-Driven Airship

The structure of a buoyancy-driven airship is shown in Fig. 1. This kind of airship moves forward by a cyclic change of the net lift of the craft and of the position of ballast. The detail of the mechanism of operating and the traditional modelling way have already been given in some papers Purandare [2007], Wu, Moog et Hu. [2009], Wu, Moog, Martinez et Hu. [2009].

The paper is organized as follows. Section 2 is devoted to the modelling and control of the most elementary special case of the airship, which represents the airship subject to three 
assumptions. The airship is liberated in Section 3 by dropping a assumption that the center of the volume $\mathrm{O}$ is fixed. In this case, the center of mass of the overall system is subject to a ballistic motion and the controllable subsystem remains similar to the previous elementary system. In Section 4, the airship model is completed with the influence of suitable added masses and Section 5 displays the full model including the aerodynamic forces.

\section{THE AIRSHIP WITH FIXED CENTER OF VOLUME}

To derive an elementary special case of the complex airship, the dynamics of the airship are not only restricted to the vertical plane, but it is also assumed that the center of volume $\mathrm{O}$ of the airship is fixed, which means that the airship can only rotate round $\mathrm{O}$, but the longitudinal and horizontal translation velocities $v_{3}$ and $v_{1}$ are zero. It also assumes that there is no inertial force and no aerodynamic force acting on the vehicle.

These assumptions reduce to:

\section{Assumptions:}

$\mathbf{A}_{\mathbf{3}}$ : The center of the volume $\mathrm{O}$ is fixed, the airship have no translational velocities.

$\mathbf{A}_{2}$ : No fluid inertial force.

$\mathbf{A}_{\mathbf{1}}$ : No aerodynamic force.

A mathematic model is derived as follows.

\subsection{Modelling}

Under the situation described above, the airship rotates around $\mathrm{O}$ with respect to the forward and backward movements of the ballast, as shown in Fig 2 and 3, but $\mathrm{O}$ does not have any translation velocities.

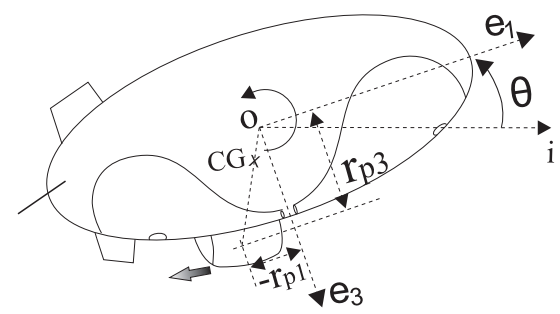

Fig. 2. Positive rotation

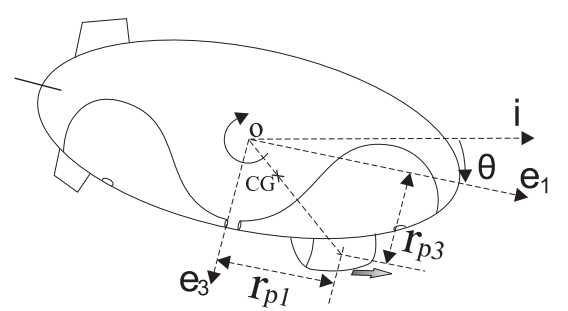

Fig. 3. Negative rotation

In this case, the airship is similar to a double pendulum as described in Fig. 4. The rotational joint at point $\mathrm{O}$ is not actuated. The joint between the two links of the double pendulum is prismatic and actuated. The double pendulum, inverted or not, has been considered as a classical control example in many references Wie [1998].

Here, the body of the airship except the ballast is denoted by the link 1 which is fixed in the point $\mathrm{O}$, and the ballast is denoted

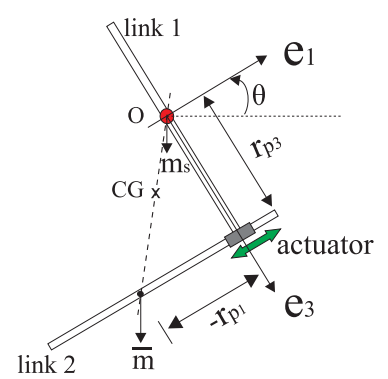

Fig. 4. The airship system with a fixed point

by another link which can be moved by a actuator. The position of the center of the mass of the ballast in the non-inertial frame in the vertical plane is $\left(r_{p 1}, r_{p 3}\right)$. The system can swing along with the actuator changing the position of $\bar{m}$, which is as same as the operation of the airship. Here, $r_{p 3}$ is constant.

The lagrangian of that simplified airship system is

$$
L=\frac{1}{2} J \dot{\theta}^{2}+\frac{1}{2} m \bar{v}_{m}^{2}-\bar{m} g\left(r_{p 3} \cos \theta-r_{p 1} \sin \theta\right) .
$$

With the Lagrangian, the equations of motion can easily be found as,

$$
\begin{aligned}
\ddot{\theta}= & \zeta_{1}=-\frac{1}{J+\bar{m} r_{p 1}^{2}}\left(\bar{m} r_{p 3} r_{p 1} \dot{\theta}^{2}+2 \bar{m} r_{p 1} \dot{r}_{p 1} \dot{\theta}\right. \\
& \left.+\bar{m} g r_{p 1} \cos \theta+r_{p 3} u\right) \\
\ddot{r}_{p 1}= & \sigma_{1}=\frac{1}{J+\bar{m} r_{p 1}^{2}}\left(\left(J r_{p 1}+\bar{m} r_{p 1}^{3}+\bar{m} r_{p 3}^{2} r_{p 1}\right) \dot{\theta}^{2}\right. \\
& +2 \bar{m} r_{p 3} r_{p 1} \dot{r}_{p 1} \dot{\theta}-\left(J+\bar{m} r_{p 1}^{2}\right) g \sin \theta+\bar{m} g r_{p 3} r_{p 1} \cos \theta \\
& \left.+\left(\frac{J}{\bar{m}}+r_{p 1}^{2}+r_{p 3}^{2}\right) u\right)
\end{aligned}
$$

where, $u$ is the input denoted the force applied on the ballast along the $e_{1}$ axis direction.

\subsection{Maximal linearization of a minimum phase model}

Standard computation Conte et al. [2007], Isidori [1999] show that the system is fully accessible and not fully linearizable by static state feedback. It is thus interesting to look for a maximal feedback linearization and to search for output function with the largest relative degree. This is done next. Following the notations in Conte et al. [2007] the following results are obtained. According to the system (1)-(2).

$$
\begin{aligned}
& \mathcal{H}_{\infty}=0 \\
& \mathcal{H}_{3}=\operatorname{span}\left\{d \phi_{1}, d \phi_{2}\right\}
\end{aligned}
$$

and $\mathcal{H}_{4}$ is not integrable. $\mathcal{H}_{3}$ represents the codistribution which consists of all differential forms whose relative degree is at least 3. Here, $\phi_{1}$ and $\phi_{2}$ as follows,

$$
\begin{aligned}
& \phi_{1}=J \dot{\theta}+\left(r_{p 1}^{2} \dot{\theta}+r_{p 3}^{2} \dot{\theta}+r_{p 3} \dot{r}_{p 1}\right) \bar{m} \\
& \phi_{2}=\theta+\frac{r_{p 3}}{\sqrt{\frac{J}{\bar{m}}+r_{p 3}^{2}}} \arctan \frac{r_{p 1}}{\sqrt{\frac{J}{\bar{m}}+r_{p 3}^{2}}}
\end{aligned}
$$

In $\phi_{1}, J \dot{\theta}$ is the angular momentum of airship's body in the vertical plane, and $\left(r_{p 1}^{2} \dot{\theta}+r_{p 3}^{2} \dot{\theta}+r_{p 3} \dot{r}_{p 1}\right) \bar{m}$ is the ballast's angular momentum $\boldsymbol{r}_{\boldsymbol{p}} \times \bar{m} \boldsymbol{v}_{\boldsymbol{p}}$ in the vertical plane $\left(\boldsymbol{r}_{\boldsymbol{p}}\right.$ and $\boldsymbol{v}_{\boldsymbol{p}}$ are the position and velocity of the ballast in the non-inertial frame 
respectively). So, $\phi_{1}$ is the angular momentum of the whole airship around the point O. $\phi_{1}$ and $\phi_{2}$ also have the following relation,

$$
\dot{\phi}_{2}=\frac{1}{J+\left(r_{p 1}^{2}+r_{p 3}^{2}\right) \bar{m}} \phi_{1}
$$

At this stage, any combination of $\phi_{1}$ and $\phi_{2}$ has relative degree 3 and its feedback linearization will yield a linear controllable subsystems of dimension 3 with a one dimensional zero dynamics. The following result shows the possibility to ensure that the system is minimum phase which has a decisive impact on the doability of this control design.

Theorem 1: $\phi_{1}+k \phi_{2}$ has stable zero dynamics for $k>0$.

Proof: Under this situation, new states are chosen as follows

$$
\begin{aligned}
& \xi_{1}=\phi_{1}+k \phi_{2} \\
& \xi_{2}=\dot{\phi}_{1}+k \dot{\phi}_{2} \\
& \xi_{3}=\ddot{\phi}_{1}+k \ddot{\phi}_{2} \\
& \xi_{4}=\phi_{1}
\end{aligned}
$$

From the zero dynamics of $\xi_{2}, \dot{\phi}_{1}+k \dot{\phi}_{2}=0$, so $\dot{\phi}_{1}=-k \dot{\phi}_{2}$, and because of the relation (3). The zero dynamics of $\xi_{4}$ as follows,

$$
\dot{\xi}_{4}=\dot{\phi}_{1}=-k \dot{\phi}_{2}=-k \frac{1}{J+\left(r_{p 1}^{2}+r_{p 3}^{2}\right) \bar{m}} \phi_{1}
$$

So, for $k>0$, the system is asymptotically stable.

\subsection{Simulations}

The family of outputs $y=\phi_{1}+k \phi_{2}$ for a varying real number $k$ is considered now. The special case (1)-(2) is stabilized through standard input-output linearization according to section 2.2. From Theorem 1, it is mandatory to pick $k>0$ to ensure internal stability of the closed loop system. Its actual value is a tuning parameter which influences the velocity of the zero dynamics. Consider the following desired error equation:

$$
y^{(3)}+\lambda_{2} y^{(2)}+\lambda_{1} y^{(1)}+\lambda_{0}\left(y-y_{e}\right)=0
$$

here, $y_{e}$ is the desired value for $y$. The parameters $k=50$, $\lambda_{2}=2, \lambda_{1}=2, \lambda_{0}=1$.

Fig. 5 and 6 are simulating results of the dynamics of the $\theta, r_{p 1}$ and the control input $u$ under initial errors. From this result, $\theta$ and $r_{p 1}$ stabilize at the equilibrium after 30 s.
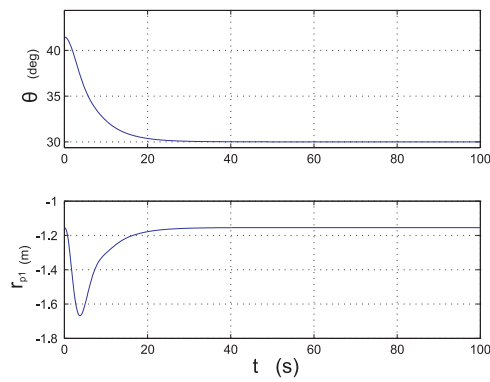

Fig. 5. Dynamics of $\theta$ and $r_{p 1}$ with initial error

\section{THE AIRSHIP WITH LIBERATED CENTER POINT}

Generally, aircrafts rotate around the center of the mass, not the center of the volume, like helicopters. In the first elementary

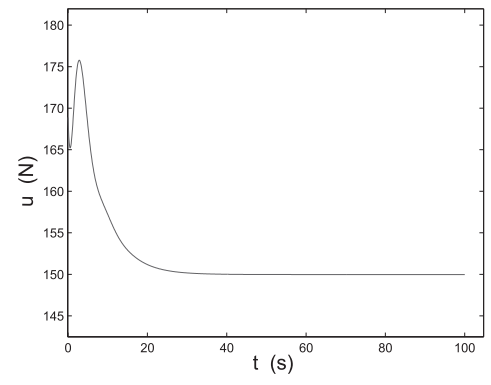

Fig. 6. The behavior of input $u$

special case, the aircraft is assumed that it rotates around the center of the volume $\mathrm{O}$. So by liberating the pivot $\mathrm{O}$, in other words, the pivot $\mathrm{O}$ can move freely in the vertical plane, the model is further close to the complete airship model.

Under this situation, the airship does not rotate around $\mathrm{O}$ any more, and it rotates around the center of the gravity $\mathrm{CG}$. But the non-inertial frame is still attached at point $\mathrm{O}$.

In this case, $A_{3}$ is dropped and we have the following assumptions.

\section{Assumptions:}

$\mathbf{A}_{\mathbf{2}}$ : No fluid inertial force.

$\mathbf{A}_{1}$ : No aerodynamic force.

\subsection{Modelling}

Newton-Law is used to develop the mathematic model futher. In the inertial frame, the total external force and moment applied on the airship are $m_{0} g \boldsymbol{k}$ and $\bar{m} g \boldsymbol{r}_{\boldsymbol{s}} \times \boldsymbol{k}$, where, $m_{0}$ is the net lift, and $\boldsymbol{k}$ is a unit vector along the direction of gravity. So the total external force in the non-inertial frame is,

$$
\begin{aligned}
\boldsymbol{F}_{\text {total }} & =m_{0} g \boldsymbol{R}_{1}^{T} \boldsymbol{k} \\
\boldsymbol{M}_{\text {total }} & =\bar{m} g \boldsymbol{R}_{1}^{T} \boldsymbol{r}_{s} \times \boldsymbol{k}
\end{aligned}
$$

The total external force also can be denoted as follows $\mathrm{Wu}$, Moog et Hu. [2009],

$$
\boldsymbol{F}_{\text {total }}=\boldsymbol{\Omega} \times \boldsymbol{B}+\boldsymbol{R}^{T} \boldsymbol{f}_{\text {total }}=\boldsymbol{\Omega} \times\left(m_{s} \boldsymbol{v}+\boldsymbol{B}_{\boldsymbol{p}}\right)+m_{s} \dot{\boldsymbol{v}}+\boldsymbol{u}
$$

where, $m_{s} \dot{\boldsymbol{v}}$ and $\boldsymbol{u}$ are the total external force on the body of the airship and the ballast respectively. $\boldsymbol{\Omega} \times \boldsymbol{B}$ or $\boldsymbol{\Omega} \times\left(m_{s} \boldsymbol{v}+\boldsymbol{B}_{\boldsymbol{p}}\right)$ are coriolis forces.

Similarly, the total external moment can be denoted as follows,

$$
\begin{aligned}
& \boldsymbol{M}_{\text {total }}=\boldsymbol{\Omega} \times \Pi+\boldsymbol{v} \times \boldsymbol{B}+\boldsymbol{R}^{\boldsymbol{T}} \tau_{\text {total }} \\
&=\boldsymbol{\Omega} \times\left(\boldsymbol{r}_{\boldsymbol{p}} \times \boldsymbol{B}_{\boldsymbol{p}}+\boldsymbol{J}_{s} \boldsymbol{\Omega}\right)+\left(\boldsymbol{v} \times m_{s} \boldsymbol{v}+\left(\boldsymbol{v}+\dot{\boldsymbol{r}}_{\boldsymbol{p}}\right) \times \boldsymbol{B}_{\boldsymbol{p}}\right) \\
&+\left(\boldsymbol{J}_{s} \dot{\boldsymbol{\Omega}}+\boldsymbol{r}_{\boldsymbol{p}} \times \boldsymbol{u}\right)
\end{aligned}
$$

where, $\boldsymbol{\Omega} \times \Pi=\boldsymbol{\Omega} \times\left(\boldsymbol{r}_{p} \times \boldsymbol{B}_{p}+\boldsymbol{J}_{s} \boldsymbol{\Omega}\right)$ is the torque of the centrifugal force, $\boldsymbol{v} \times \boldsymbol{B}=\boldsymbol{v} \times m_{s} \boldsymbol{v}+\left(\boldsymbol{v}+\dot{\boldsymbol{r}}_{\boldsymbol{p}}\right) \times \boldsymbol{B}_{\boldsymbol{p}}$ is the torque of the coriolis force. $\left(\boldsymbol{J}_{\boldsymbol{s}} \dot{\boldsymbol{\Omega}}+\boldsymbol{r}_{\boldsymbol{p}} \times \boldsymbol{u}\right)$ is the torque respect to the non-inertial frame.

From the relations (9) and (21), (10) and (22), the $\dot{\boldsymbol{v}}$ and $\dot{\boldsymbol{\Omega}}$ are derived as,

$$
\begin{aligned}
\dot{\boldsymbol{v}}= & m_{s}^{-1}\left(\left(m_{s} \boldsymbol{v}+\boldsymbol{B}_{p}\right) \times \mathbf{\Omega}+m_{0} g \boldsymbol{R}_{\mathbf{1}}^{\boldsymbol{T}} \boldsymbol{k}-\boldsymbol{u}\right) \\
\dot{\boldsymbol{\Omega}}= & \boldsymbol{J}_{s}^{-\mathbf{1}}\left(\left(\boldsymbol{J}_{s} \mathbf{\Omega}+\boldsymbol{r}_{\boldsymbol{p}} \times \boldsymbol{B}_{p}\right) \times \mathbf{\Omega}+\mathbf{\Omega} \times \boldsymbol{r}_{\boldsymbol{p}} \times \boldsymbol{B}_{p}\right. \\
& \left.+\bar{m} g \boldsymbol{r}_{\boldsymbol{p}} \times \boldsymbol{R}_{\mathbf{1}}^{\boldsymbol{T}} \boldsymbol{k}-\boldsymbol{r}_{\boldsymbol{p}} \times \boldsymbol{u}\right)
\end{aligned}
$$


where, $\boldsymbol{B}_{\boldsymbol{p}}=\bar{m} \boldsymbol{v}_{\boldsymbol{p}}=\bar{m}\left(\boldsymbol{v}+\dot{\boldsymbol{r}}_{\boldsymbol{p}}+\boldsymbol{\Omega} \times \boldsymbol{r}_{\boldsymbol{p}}\right)$ is the momentum of the ballast.

From (13) and (14), the mathematic model in the vertical plane is as follows,

$$
\begin{aligned}
\ddot{\theta} & =\zeta_{2}=\zeta_{1}+\frac{\left(\dot{v_{3}}-\dot{\theta} v_{1}\right) \bar{m} r_{p 1}}{J+\bar{m} r_{p 1}^{2}} \\
\ddot{r}_{p 1} & =\sigma_{2}=\sigma_{1}+\frac{\bar{m} r_{p 1} r_{p 3}\left(\dot{\theta} v_{1}-\dot{v}_{3}\right)-\left(J+\bar{m} r_{p 1}^{2}\right)\left(\dot{\theta} v_{3}+\dot{v}_{1}\right)}{J+\bar{m} r_{p 1}^{2}} \\
\dot{v}_{1} & =\varkappa_{1}=\frac{1}{m_{s}}\left(-m_{s} \dot{\theta} v_{3}+\left(\bar{m}-m_{0}\right) g \sin \theta-u\right) \\
\dot{v}_{3} & =\varkappa_{3}=\frac{1}{m_{s}+\bar{m}}\left(\left(m_{s}+\bar{m}\right) \dot{\theta} v_{1}+m_{0} g \cos \theta+\bar{m} r_{p 3} \dot{\theta}^{2}\right. \\
& \left.+2 \bar{m} \dot{\theta} \dot{r}_{p 1}+\bar{m} r_{p 1} \ddot{\theta}\right)
\end{aligned}
$$

Comparing the elementary special case (1)-(2) with model (15)(18), It's easy to find that the elementary case (1)-(2) is a subsystem of the model (15)-(18), which can be shown by the simulation.

\subsection{Maximal linearization of a minimum phase model}

System (15)-(18) is now subject to a ballistic motion (of point CG) and to be out of the action of the control input. In other words, there exist some non-controllable states besides the controllable or accessible system. Again, following standard computation Conte et al. [2007], one computers the noncontrollable subsystem whose coordinates are denoted by $\psi_{1}$ and $\psi_{2}$ :

where

$$
\mathcal{H}_{\infty}=\operatorname{span}\left\{d \psi_{1}, d \psi_{2}\right\}
$$

$$
\begin{aligned}
& \psi_{1}=\left(r_{p 1} \dot{\theta}-\frac{m_{s}+\bar{m}}{\bar{m}} v_{3}\right)^{2}+\left(\dot{r}_{p 1}+r_{p 3} \dot{\theta}+\frac{m_{s}+\bar{m}}{\bar{m}} v_{1}\right)^{2} \\
& \psi_{2}=\left(r_{p 1} \dot{\theta}-\frac{m_{s}+\bar{m}}{\bar{m}} v_{3}\right) \cos \theta+\left(\dot{r}_{p 1}+r_{p 3} \dot{\theta}+\frac{m_{s}+\bar{m}}{\bar{m}} v_{1}\right) \sin \theta
\end{aligned}
$$

$\left(m_{s}+\bar{m}\right) \psi_{1}$ is the kinetic energy of CG, and $\psi_{2}$ denotes the horizontal velocity of CG, which means that the airship includes the ballistic motion of the center of gravity CG. Fig. 9 displays this ballistic motion.

Thus the 6-dimensional state system (15)-(18) can be decoupled into a 2-dimensional non-controllable subsystem and a 4dimensional subsystem whose structural properties are similar to those of model (1)-(2).

where,

$$
\mathcal{H}_{3}=\operatorname{span}\left\{d \tilde{\phi}_{1}, d \tilde{\phi}_{2}\right\} \oplus \mathcal{H}_{\infty}
$$

$$
\begin{aligned}
& \tilde{\phi}_{1}=J \dot{\theta}+\left(r_{p 1}^{2} \dot{\theta}+r_{p 3}^{2} \dot{\theta}+r_{p 3} \dot{r}_{p 1}\right) \frac{\bar{m} m_{s}}{\bar{m}+m_{s}} \\
& \tilde{\phi}_{2}=\theta+\frac{r_{p 3}}{\sqrt{\frac{\bar{m}+m_{s}}{\bar{m} m_{s}}} J+r_{p 3}^{2}} \arctan \frac{r_{p 1}}{\sqrt{\frac{\bar{m}+m_{s}}{\bar{m} m_{s}}} J+r_{p 3}^{2}}
\end{aligned}
$$

Here, $\tilde{\phi}_{1}$ is the angular momentum of the airship around the center of the gravity CG as previous. The relation of $\tilde{\phi}_{1}$ and $\tilde{\phi}_{1}$ is as follows,

$$
\dot{\tilde{\phi}}_{2}=\frac{1}{J+\left(r_{p 1}^{2}+r_{p 3}^{2}\right) \frac{\bar{m} m_{s}}{\bar{m}+m_{s}}} \tilde{\phi}_{1}
$$

Theorem 2: $\tilde{\phi}_{1}+\tilde{k} \tilde{\phi}_{2}$ has stable zero dynamics for $\tilde{k}>0$.

The way to prove Theorem $\mathbf{2}$ is the same as in the previous section.

\subsection{Simulations}

The control law and the values of the parameters here is the same as the previous part. The initial values of the states are $\theta_{0}=41.5, r_{p 10}=-1.15 \mathrm{~m}, v_{10}=1.8 \mathrm{~m} / \mathrm{s}$ and $v_{30}=0$, and also $m_{0}=1 \mathrm{~kg}, m_{s}=269 \mathrm{~kg}$.

The similar performances of the controller are got,see fig. 7-8.

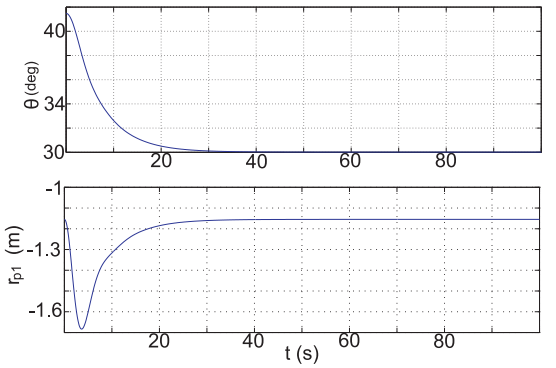

Fig. 7. Dynamics of $\theta$ and $r_{p 1}$ with initial error

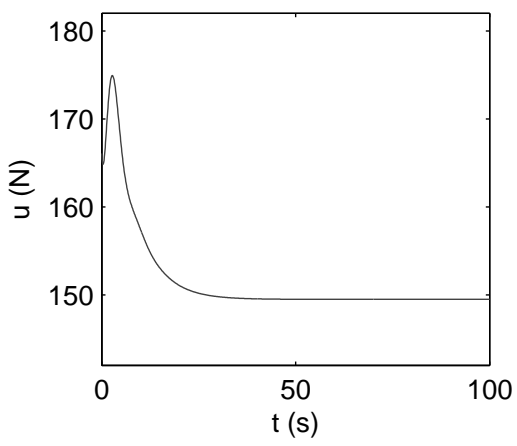

Fig. 8. The behavior of input $u$

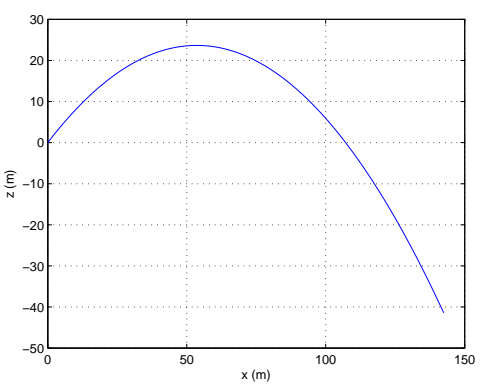

Fig. 9. Ballistic motion of CG

\section{THE AIRSHIP WITH LIBERATED CENTER AND ADDED MASSES}

The inertia of airships with a large Volume/Mass ratio is much more significant in comparison with conventional airplanes, which must be considered. Added mass is designed to represent some of there initial forces.

Assumption $\mathbf{A}_{2}$ is dropped. But aerodynamic forces are still ignored. 


\section{Assumptions:}

$\mathbf{A}_{\mathbf{1}}$ : No aerodynamic force.

In this section, the role of the result of previous sections is shown.

\subsection{Modelling}

The matrix $\boldsymbol{M}_{\boldsymbol{a d d}}$ including these added masses is called the inertia matrix $\mathrm{Wu}, \mathrm{Moog}$ et $\mathrm{Hu}$. [2009].

$$
\boldsymbol{M}_{\text {add }}=\left(\begin{array}{c|c}
\boldsymbol{M}_{\boldsymbol{f}} & * \\
\hline * & \boldsymbol{J}_{f}
\end{array}\right)
$$

$\boldsymbol{M}_{\boldsymbol{f}}$ is the symmetric $3 \times 3$ added mass matrix; $\boldsymbol{J}_{\boldsymbol{f}}$ is the added inertia matrix. $\boldsymbol{M}_{\boldsymbol{f}}=\operatorname{diag}\left\{\begin{array}{llll}m_{11} & m_{22} & m_{33}\end{array}\right\}$ and $\boldsymbol{J}_{\boldsymbol{f}}=$ $\operatorname{diag}\left\{m_{44} m_{55} m_{66}\right\}$.

In an ideal fluid, the kinetic energy $T_{a d d}$ of fluid inertia is

$$
\begin{aligned}
T_{a d d}= & \frac{1}{2} \sum_{i=1}^{6} \sum_{j=1}^{6} m_{i j} \zeta_{i} \zeta_{j}=\frac{1}{2}\left(m_{11} v_{1}^{2}+m_{22} v_{2}^{2}\right. \\
& \left.+m_{33} v_{3}^{2}+m_{44} \Omega_{1}^{2}+m_{55} \Omega_{2}^{2}+m_{66} \Omega_{3}^{2}\right)
\end{aligned}
$$

here $\zeta_{1}=v_{1}, \zeta_{2}=v_{2}, \zeta_{3}=v_{3}, \zeta_{4}=\Omega_{1}, \zeta_{5}=\Omega_{2}, \zeta_{6}=\Omega_{3}$. The momenta $\boldsymbol{B}=\left(B_{1}, B_{2}, B_{3}\right)^{T}$ and moments of momentum $\boldsymbol{K}=\left(B_{4}, B_{5}, B_{6}\right)^{T}$ of fluid inertia are related to the kinetic energy $T_{\text {add }}$ :

$$
B_{i}=\frac{\partial T_{a d d}}{\partial \zeta_{i}} \quad(i=1,2, \cdots, 6)
$$

So,

$$
\begin{aligned}
& \boldsymbol{B}=\boldsymbol{M}_{\boldsymbol{f}} \boldsymbol{v} \\
& \boldsymbol{K}=\boldsymbol{J}_{\boldsymbol{f}} \boldsymbol{\Omega}
\end{aligned}
$$

So, the inertial forces $\boldsymbol{F}_{\boldsymbol{I}}$ and moments $\boldsymbol{M}_{\boldsymbol{I}}$ acting on the airship are as follows

$$
\begin{aligned}
\boldsymbol{F}_{\boldsymbol{I}}= & -\frac{d \boldsymbol{B}}{d t}=-\left(\frac{d \tilde{\boldsymbol{B}}}{d t}+\boldsymbol{\Omega} \times \boldsymbol{B}\right) \\
& =-\boldsymbol{M}_{f} \dot{\boldsymbol{v}}+\boldsymbol{M}_{f} \boldsymbol{v} \times \boldsymbol{\Omega} \\
\boldsymbol{M}_{\boldsymbol{I}}= & -\frac{d \boldsymbol{K}}{d t}=-\left(\frac{d \tilde{\boldsymbol{K}}}{d t}+\boldsymbol{\Omega} \times \boldsymbol{K}+\boldsymbol{v} \times \boldsymbol{B}\right) \\
& =-\boldsymbol{J}_{f} \dot{\boldsymbol{\Omega}}+\boldsymbol{J}_{f} \boldsymbol{\Omega} \times \boldsymbol{\Omega}+\boldsymbol{M}_{\boldsymbol{f}} \boldsymbol{v} \times \boldsymbol{v}
\end{aligned}
$$

here, $\frac{d \boldsymbol{B}}{d t}, \frac{d \boldsymbol{K}}{d t}$ denote the time-derivative of momentum $\boldsymbol{B}$ and angular momentum $\boldsymbol{K}$ with respect to the inertial frame, $\frac{d \tilde{\boldsymbol{B}}}{d t}$ and $\frac{d \tilde{\boldsymbol{K}}}{d t}$ denote the time-derivative in the body frame.

Comparing with the previous case (9)-(10), the total external force should include inertial forces as follows,

$$
\begin{aligned}
\boldsymbol{F}_{\text {total }} & =m_{0} g \boldsymbol{R}_{1}^{T} \boldsymbol{k}+\boldsymbol{F}_{\boldsymbol{I}} \\
\boldsymbol{M}_{\text {total }} & =\bar{m} g \boldsymbol{R}_{1}^{T} \boldsymbol{r}_{s} \times \boldsymbol{k}+\boldsymbol{M}_{\boldsymbol{I}}
\end{aligned}
$$

Let $m_{1}=m_{s}+m_{11}, m_{3}=m_{s}+m_{33}$ and $J_{2}=J+J_{22}$, the model of the airship with the effects of the inertial forces is as follows,

$$
\begin{aligned}
\ddot{\theta} & =\zeta_{3}=\zeta_{2}^{\prime}+\frac{\left(m_{3}-m_{1}\right) v_{1} v_{3}}{J_{2}+\bar{m} r_{p 1}^{2}} \\
\ddot{r}_{p 1} & =\sigma_{3}=\sigma_{2}^{\prime}+\frac{\left(m_{3}-m_{1}\right) v_{1} v_{3}}{J_{2}+\bar{m} r_{p 1}^{2}} r_{p 3} \\
\dot{v}_{1} & =\varkappa_{1}^{\prime} \\
\dot{v}_{3} & =\varkappa_{3}^{\prime}
\end{aligned}
$$

Note that model (15)-(18) reduces to the special case of model (23)-(23) if $m_{11}=m_{11}=J_{22}=0$ (no added mass).

In the fluid inertial forces, $\left(m_{3}-m_{1}\right) v_{1} v_{3}$ is pitching moment, which will be included in the aerodynamic forces. This is because when measuring the aerodynamic forces of the airship by a wind tunnel test, a part of fluid inertial forces, for instance $\left(m_{3}-m_{1}\right) v_{1} v_{3}$, is already included in the results of wind tunnel test, which is behaved as aerodynamic forces. So, $\left(m_{3}-m_{1}\right) v_{1} v_{3}$ be ignored in the above model Ouyang [2003]. Then, it is as,

$$
\begin{aligned}
\ddot{\theta} & =\zeta_{3}=\zeta_{2}^{\prime} \\
\ddot{r}_{p 1} & =\sigma_{3}=\sigma_{2}^{\prime} \\
\dot{v}_{1} & =\varkappa_{1}^{\prime} \\
\dot{v}_{3} & =\varkappa_{3}^{\prime}
\end{aligned}
$$

All $J$ and $m_{s}$ in (15)-(18) change into $J_{2}, m_{1}$ and $m_{3}$ as follows, other items keep unchanged.

where

$$
\begin{aligned}
\zeta_{2}^{\prime} & =-\frac{1}{J_{2}+\bar{m} r_{p 1}^{2}}\left(\bar{m} r_{p 3} r_{p 1} \dot{\theta}^{2}+2 \bar{m} r_{p 1} \dot{r}_{p 1} \dot{\theta}+\bar{m} g r_{p 1} \cos \theta\right. \\
& \left.+r_{p 3} u-\left(\dot{v_{3}}-\dot{\theta} v_{1}\right) \bar{m} r_{p 1}\right) \\
\sigma_{2}^{\prime} & =\frac{1}{J_{2}+\bar{m} r_{p 1}^{2}}\left(\left(J_{2} r_{p 1}+\bar{m} r_{p 1}^{3}+\bar{m} r_{p 3}^{2} r_{p 1}\right) \dot{\theta}^{2}+2 \bar{m} r_{p 3} r_{p 1} \dot{r}_{p 1} \dot{\theta}\right. \\
& -\left(J_{2}+\bar{m} r_{p 1}^{2}\right) g \sin \theta+\bar{m} g r_{p 3} r_{p 1} \cos \theta+\left(\frac{J_{2}}{\bar{m}}+r_{p 1}^{2}+r_{p 3}^{2}\right) u \\
& \left.+\bar{m} r_{p 1} r_{p 3}\left(\dot{\theta} v_{1}-\dot{v}_{3}\right)-\left(J_{2}+\bar{m} r_{p 1}^{2}\right)\left(\dot{\theta} v_{3}+\dot{v}_{1}\right)\right) \\
\varkappa_{1}^{\prime} & =\frac{-m_{3} \dot{\theta} v_{3}+\left(\bar{m}-m_{0}\right) g \sin \theta-u}{m_{1}} \\
\varkappa_{3}^{\prime} & =\frac{\left(m_{1}+\bar{m}\right) \dot{\theta} v_{1}+m_{0} g \cos \theta+\bar{m} r_{p 3} \dot{\theta}^{2}+2 \bar{m} \dot{\theta} \dot{r}_{p 1}+\bar{m} r_{p 1} \ddot{\theta}}{m_{3}+\bar{m}}
\end{aligned}
$$

\subsection{Maximal linearization of a minimum phase model}

The model (27)-(30) be decoupled into a non-controllable system and controllable one as it was done for model (15)-(18). The velocities of the center of the gravity CG are not affected by the control input, however their mathematical expressions become a bit more involved.

$$
\mathcal{H}_{\infty}=\operatorname{span}\left\{d \psi_{1}^{\prime} d \psi_{2}^{\prime}\right\}
$$

where

$$
\begin{aligned}
& \psi_{1}=\left(r_{p 1} \dot{\theta}-\frac{m_{3}+\bar{m}}{\bar{m}} v_{3}\right)^{2}+\left(\dot{r}_{p 1}+r_{p 3} \dot{\theta}+\frac{m_{1}+\bar{m}}{\bar{m}} v_{1}\right)^{2} \\
& \psi_{2}=\left(r_{p 1} \dot{\theta}-\frac{m_{3}+\bar{m}}{\bar{m}} v_{3}\right) \cos \theta+\left(\dot{r}_{p 1}+r_{p 3} \dot{\theta}+\frac{m_{1}+\bar{m}}{\bar{m}} v_{1}\right) \sin \theta
\end{aligned}
$$

Surprisingly, there is now only one single independent function $\widetilde{\phi}_{2}^{\prime}$ which has relative degree 3 for the model (27)-(30). More precisely, compute $\mathcal{H}_{3}$ : 


$$
\mathcal{H}_{3}=\operatorname{span}\left\{d \tilde{\phi}_{2}^{\prime} \omega\right\} \oplus \mathcal{H}_{\infty}
$$

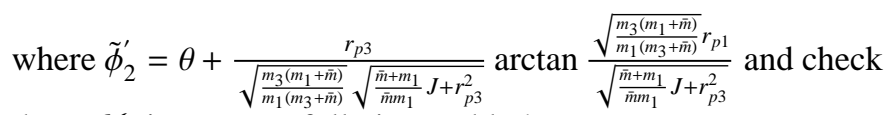
those $\mathcal{H}_{3}$ is no more fully integrable !

Compute $\mathcal{H}_{2}$ as:

$$
\mathcal{H}_{2}=\operatorname{span}\left\{y_{1}^{\prime} \quad y_{2}^{\prime} \quad \tilde{\phi}_{1}^{\prime}\right\} \oplus \mathcal{H}_{\infty}
$$

where

$$
\begin{aligned}
& y_{1}^{\prime}=\frac{\bar{m}}{m_{3}+\bar{m}} r_{p 1} \dot{\theta}-v_{3} \\
& y_{2}^{\prime}=\frac{\bar{m}}{m_{1}+\bar{m}}\left(\dot{r}_{p 1}+r_{p 3} \dot{\theta}\right)+v_{1} \\
& \tilde{\phi}_{1}^{\prime}=\left(\frac{\bar{m}+m_{1}}{\bar{m} m_{1}} J+\frac{m_{3}\left(m_{1}+\bar{m}\right)}{m_{1}\left(m_{3}+\bar{m}\right)} r_{p 1}^{2}+r_{p 3}^{2}\right) \dot{\theta}+r_{p 3} \dot{r}_{p 1}
\end{aligned}
$$

As the previous sections, one has the relation involving the angular momentum,

$$
\dot{\tilde{\phi}}_{2}^{\prime}=\frac{1}{\frac{\bar{m}+m_{1}}{\bar{m} m_{1}} J+\frac{m_{3}\left(m_{1}+\bar{m}\right)}{m_{1}\left(m_{3}+\bar{m}\right)} r_{p 1}^{2}+r_{p 3}^{2}} \tilde{\phi}_{1}^{\prime}
$$

Despite these diametric changes with section 3 , it is now argued that the control scheme which has been computed for the elementary special case in the section 2 is still valid, thanks to some approximation. This is done next!

\subsection{Simulations}

Since $\tilde{\phi}_{1}^{\prime}$ does not have relative degree 3 anymore as $\tilde{\phi}_{2}^{\prime}$, the Theorem $1 \& 2$ are not true. But fortunately, the second-time derivative of $\tilde{\phi}_{1}^{\prime}$ has a small coefficient of $u$, so it is proposed to neglect this coefficient.

More precisely, define again the output $y=\tilde{\phi}_{1}^{\prime}+k \tilde{\phi}_{2}^{\prime}$, for some $k>0$.

Instead of the coordinates (4)-(7), define

$$
\begin{aligned}
& \zeta_{1}=\tilde{\phi}_{1}^{\prime}+k \tilde{\phi}_{2}^{\prime} \\
& \zeta_{2}=\dot{\tilde{\phi}}_{1}^{\prime}+k \dot{\tilde{\phi}}_{2}^{\prime} \\
& \zeta_{3}=\ddot{\tilde{\phi}}_{1}^{\prime}+k \ddot{\tilde{\phi}}_{2}^{\prime}-\Delta u \\
& \zeta_{4}=\tilde{\phi}_{1}^{\prime}
\end{aligned}
$$

where $\ddot{\widetilde{\phi}}_{1}^{\prime}=\Pi+\Delta u$

Instead of solving (8), consider the following equation:

$$
\dot{\zeta}_{3}+\lambda_{2} \zeta_{3}+\lambda_{1} y^{(1)}+\lambda_{0}\left(y-y_{e}\right)=0
$$

Note that equation (35) is strictly the equation (8) only if $\Delta \equiv 0$. In the following when $\Delta$ is small, then (35) is an approximation of (8).

Solving $u$ in the equation (35) yields the desired states feedback which is applied now to the model (27)-(30). The corresponding simulation results are displayed accordingly and show that the stability property is unchanged despite the above approximation.

Here, $m_{1}=400 \mathrm{~kg}, m_{3}=500 \mathrm{~kg}$ and the other parameters have the same values as the previous. Good control performances have been got as displayed in Fig 10 to 12 .
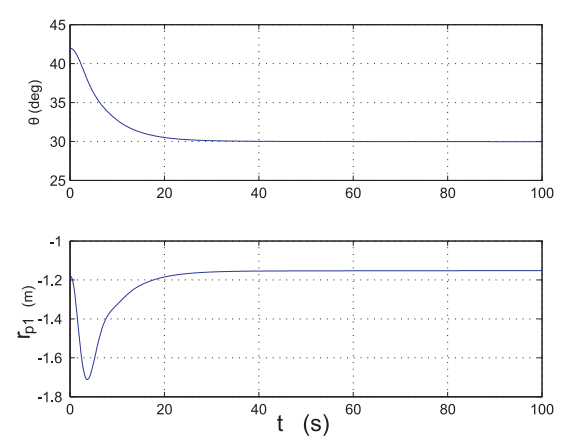

Fig. 10. Dynamics of $\theta$ and $r_{p 1}$ with initial error

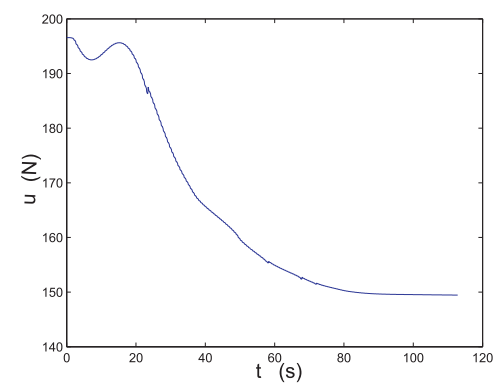

Fig. 11. The behavior of input $u$

\section{THE COMPLETE AIRSHIP MODEL}

The last step, The aerodynamic forces are added into the system, which means $\mathbf{A}_{\mathbf{1}}$ is dropped. So the following total external forces and momentums should include aerodynamic forces $\boldsymbol{F}_{\boldsymbol{a}}$ and momentums $\boldsymbol{M}_{\boldsymbol{a}}$.

$$
\begin{aligned}
\boldsymbol{F}_{\text {total }} & =m_{0} g \boldsymbol{R}_{\mathbf{1}}^{T} \boldsymbol{k}+\boldsymbol{F}_{\boldsymbol{I}}+\boldsymbol{F}_{\boldsymbol{a}} \\
\boldsymbol{M}_{\text {total }} & =m_{0} g \boldsymbol{R}_{\mathbf{1}}^{T} \boldsymbol{r}_{s} \times \boldsymbol{k}+\boldsymbol{M}_{\boldsymbol{I}}+\boldsymbol{M}_{\boldsymbol{a}}
\end{aligned}
$$

Combined (36) and (37) and, given the complete model as the follows,

$$
\begin{aligned}
\ddot{\theta} & =\zeta_{3}+\frac{M_{a}}{J+\bar{m} r_{p 1}^{2}} \\
\ddot{r}_{p 1} & =\sigma_{3}+\frac{M_{a} r_{p 3}}{J+\bar{m} r_{p 1}^{2}} \\
\dot{v}_{1} & =\varkappa_{1}^{\prime}+\frac{F_{a 1}}{m_{s}} \\
\dot{v}_{3} & =\varkappa_{3}^{\prime}+\frac{F_{a 3}}{m_{s}+\bar{m}}
\end{aligned}
$$

where,

$$
\begin{aligned}
F_{a 1} & =-X_{a} \cos \alpha+Z_{a} \sin \alpha \\
F_{a 3} & =-X_{a} \sin \alpha-Z_{a} \cos \alpha \\
X_{a} & =\frac{1}{2} \rho_{a} \nabla^{2 / 3} \boldsymbol{v}^{2}\left(C_{x 0}+C_{x}^{\alpha} \alpha^{2}\right) \\
Z_{a} & =\frac{1}{2} \rho_{a} \nabla^{2 / 3} \boldsymbol{v}^{2}\left(C_{z 0}+C_{z}^{\alpha} \alpha\right) \\
M_{a} & =\frac{1}{2} \rho_{a} \nabla \boldsymbol{v}^{2}\left(C_{m 0}+C_{m}^{\alpha} \alpha\right)
\end{aligned}
$$

$X_{a}, Z_{a}$ are aerodynamic forces along $e_{1}$ and $e_{3}$ axes, and $M_{a}$ is momentum around $e_{2}$ axis. $\alpha$ is the attack angle. 


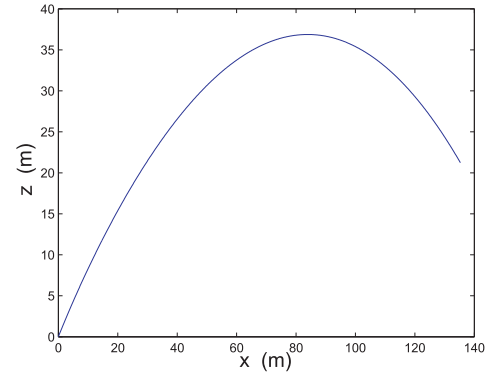

Fig. 12. Ballistic motion of CG

Compute $\mathcal{H}_{2}$ as:

$$
\begin{gathered}
\mathcal{H}_{2}=\operatorname{span}\left\{d y_{1}^{\prime} d y_{2}^{\prime} d \tilde{\phi}_{1}^{\prime} d \theta ; d r_{p 1}\right\} \\
\mathcal{H}_{3}=\operatorname{span}\left\{d \tilde{\phi}_{2}^{\prime} \omega\right\}
\end{gathered}
$$

Here, $d \tilde{\phi}_{1}^{\prime}$ and $d \tilde{\phi}_{2}^{\prime}$ have the same form as the previous sections, but the values are different. By the same way as the section 4 , a advanced nonlinear controller can be derived. The simulation results of this nonlinear controller for the complete system will be presented elsewhere.

\section{CONCLUSION}

In this paper, a new way to set up the model for a complex system is denoted. What's more important is that based on that modelling-from the fundamental and elementary mechanical structure to the complex and complete model-a nonlinear controller is designed for that complex airship system. This nonlinear controller is difficult to derive directly from the complex full model.

Even though that nonlinear controller is not a precise one, some approximation has been done, the controller behaves well, which has better performances and larger stable domain of initial conditions than the previous controllers.

\section{REFERENCES}

X. Wu, C. H. Moog, Y. Hu. Modelling and linear control of a buoyancy-driven airship. The Proceeding of 7th Asian Control Conf., pages 75-80, Hong Kong, August 2009.

X. Wu, C. H. Moog, L.A. MARQUEZ MARTINEZ and Y. $\mathrm{Hu}$. Nonlinear control of a buoyancy-driven airship. The Proceeding of 48th IEEE Conf. on Decision and Control, Shanghai, December 2009.

G. Conte, C. H. Moog and A.M. Perdon. Algebraic Methods for Nonlinear Control Systems-Theory and Applications, Chapter 3 and 9, 2nd Edition, Springer, 2006.

A. Isidori. Nonlinear Control Systems: An Introduction, Chapter 4, Springer, 1999.

J. Ouyang. Research on Modeling and Control of an Unmanned Airship, Dissertation for the Doctor Degree, Shanghai Jiaotong University, 2003.

R. Purandare. A buoyancy-Propelled Airship, Dissertation for the Doctor Degree of Philosophy, New Mexico State University, 2007.

N. Leonard and J. Graver. Model-based feedback control of autonomous. underwater gliders. IEEE Journal of Oceanic Engineering, Vol. 26, No. 4, October 2001.
B. Wie. Space Vehicle Dynamics and Control,AIAA, Page 43, June 1998.

S. V. B. Gomes and J. G. Ramos. Airship dynamic modeling for autonomous operation. The Proceedings of IEEE Int. Conf. on Robotics and Automation, pages 3462-3467, Leuven, May 1998. 